\title{
Editorial
}

\section{Decision Making in Support of Manufacturing Enterprise Transformation}

\author{
Albert Jones, ${ }^{1}$ Richard H. Weston, ${ }^{2,3}$ Bernard Grabot, ${ }^{4}$ and Bernard Hon ${ }^{5}$ \\ ${ }^{1}$ NIST, Gaithersburg, MD 20899, USA \\ ${ }^{2}$ MSI Research Institute, Wolfson School of Mechanical and Manufacturing Engineering, Loughborough University, \\ Loughborough LE11 3TU, UK \\ ${ }^{3}$ Department of Manufacturing and Materials Sciences, Cranfield University, Bedford MK43 OAL, UK \\ ${ }^{4}$ University of Toulouse, 31000 Toulouse, France \\ ${ }^{5}$ Department of Engineering, Liverpool University, Merseyside L69 3BX, UK
}

Correspondence should be addressed to Albert Jones; albert.jones@nist.gov

Received 13 November 2012; Accepted 13 November 2012

Copyright (c) 2013 Albert Jones et al. This is an open access article distributed under the Creative Commons Attribution License, which permits unrestricted use, distribution, and reproduction in any medium, provided the original work is properly cited.

To achieve the agility necessary to compete successfully in everchanging world markets, manufacturing enterprises of all sizes must exhibit a transformational leadership. Strategic, tactical, and operational decision making is an essential part of such leadership. The papers in this special issue seek to understand and characterize common transformational decision-making needs in manufacturing enterprises and the state of art in decision-making methods and technologies. Additionally, these papers identify gaps in those methods and technologies and propose research can fill those gaps.

In the paper titled "The integrated use of enterprise and system dynamics modelling techniques in support of business decisions" the authors use case studies to describe a new modelling methodology for analysing and managing dynamics and complexities in production systems. This methodology is based on a systematic transformation process that integrates enterprise, causal loop (CL), and system dynamics (SDs) modelling techniques. The methodology relies on the creation of CIMOSA process models, which are then converted to CL models. CL models are then structured and translated into equivalent SD models. The authors have used their methodology in a number of case study companies to (1) capture business processes, (2) model the dynamics impacting those processes, (3) analyse the impact of variations in customer demand on value realization, and (4) analyse the effect of constant sales orders on material supply, cost, and product realisation.
In the paper titled "Performance assessment of product service system from system architecture perspectives" the author focuses on a concept called Product Service System (PSS). The PSS enables the manufacturers of complex engineering products to incorporate support services into the entire product life cycle. However, the PSS design has imposed significant risks to the manufacturer not only in the manufacture of the product itself, but also in the provision of support services over a long period of time at a predetermined price. This paper analysed three case studies using case study research design approach and mapped the service elements of the case studies to the generic Complex Engineering Product Service System (CEPSS) model. By establishing the concept of capability distribution for a PSS enterprise, the capability of the CEPSS can be overlaid on the performance-based reward scheme so that decision makers evaluate options related to the business opportunities presented to them.

In the paper titled "Evaluating ethical responsibility in inverse decision support" the author addresses an ethical dilemma in "Inverse Decision Support." That dilemma arises whenever a decision support analyst (DSA) must evaluate the ethical responsibility in selecting a suboptimal alternative. This can happen, for example, when the actual decision maker requires the DSA's justification for a preconceived selection that does not correspond to the best option resulted from the professional resolution of the problem. To address this dilemma, the author proposed an extended application 
of the Analytic Hierarchy Process model. That extended application is consistent with the Inverse Decision Theory that is used extensively in medical decision making. The author conducted a survey of DSAs to assess their perspective of using the proposed extended application. The paper includes results from the survey. Those results show that (1) $80 \%$ of the respondents felt that the proposed extended application is useful and (2) 14\% of them expanded the usability of the extended application to their academic courses.

In the paper titled "Design of an integrated methodology for analytical design of complex supply chains" the authors review modelling capabilities needed to advance industry best practice regarding the analytical design of supply chains. Those capabilities include (1) the graphical representation and explicit description of key structural properties of complex supply chains, (2) behavioural exploration of dynamic properties of existing complex supply chains, (3) prediction of possible future behaviours of complex supply chains when uncertainties arise, and (4) predictive quantification about relative performances of alternative complex supply chain configurations, including risk assessments, when they are subjected to uncertainty. They then reviewed alternative means of deploying state-of-the-art modelling techniques, including enterprise modelling (EM), causal loop diagramming (CLD), and simulation modelling (SM). Previously the literature had not reported methods of coherently applying EM, CLD, and SM techniques in support of the lifecycle engineering of complex supply chains. The authors then describe their efforts to specify and develop an integrated, model-driven method of modeling and engineering complex supply chains. This method has the potential to support collective and individual decision making of managers and engineers.

In the paper titled "Multiobjective optimization of aircraft maintenance in thailand using goal programming: a decisionsupport model" the authors develop a multiobjective optimization model in order to evaluate suppliers for aircraft maintenance tasks, using goal programming. The authors have developed a two-step process. Authors use the model as a decision support tool for managing demand, by using aircraft and flight schedules to evaluate and generate aircraft maintenance requirements, including spare part lists. In step two, they develop a multi-objective optimization model by minimizing cost, minimizing lead time, and maximizing the quality under various constraints in the model. Finally, they demonstrate the model in the actual airline case study.

In the paper "Modelling framework to support decision making in manufacturing enterprises" the authors describe a novel systematic framework for creating coherent sets of integrated models that facilitate production planning and control (PPC) strategies in future reconfigurable manufacturing enterprises (MEs). The framework is the enhanced integrated modelling framework (EIMF). EIMF supports the engineering of common types of strategic, tactical, and operational processes found in many MEs. The authors use the CIMOSA enterprise modelling approach to develop a structured, model-driven, integrated approach that can support various PPC decisions. This new approach can capture and represent both static and dynamic aspects of an organization. Finally, the authors provide example application cases that show benefits in terms of lead time and cost reductions.

In the paper "Model driven integrated decision-making in manufacturing enterprises" the authors summarize decisionmaking requirements and solutions from four world-class manufacturing enterprises (MEs). They include observations on deployed methods of complexity handling that facilitate multipurpose, distributed decision making. They also report on examples of partially deficient "integrated decision making" which stem from a poor understanding of the relationship between structure and behavior. As a way of improving this understanding, the authors propose a "reference model of ME decision- making" and a new model-driven approach, which can "underpin integrated ME decision- making." The authors include an ME case study to explain how their approach can improve the integration of previously distinct planning functions. The modelling approach is particularly innovative in respect to the way it structures the coherent creation and experimental reuse of "fit for purpose" discrete event (predictive) simulation models at the multiple levels of abstraction. 


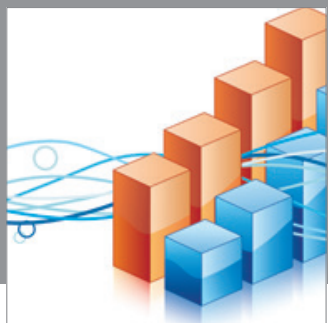

Advances in

Operations Research

mansans

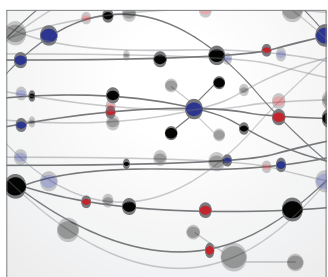

The Scientific World Journal
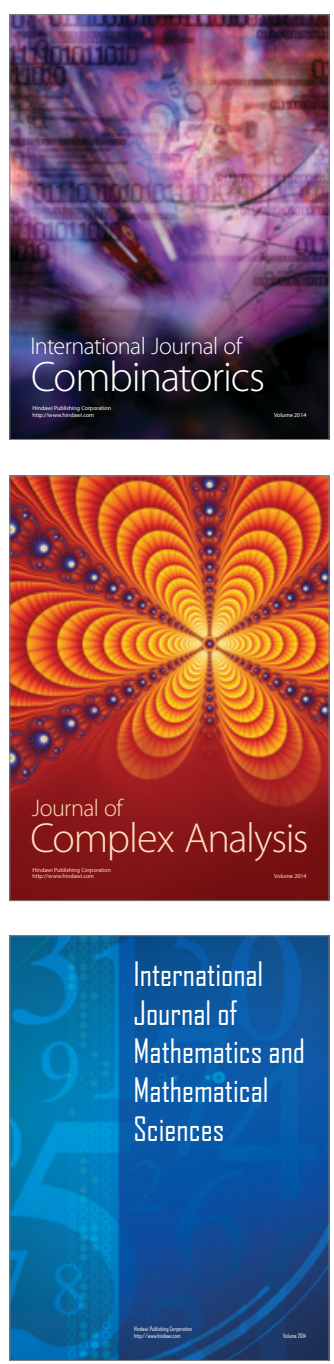
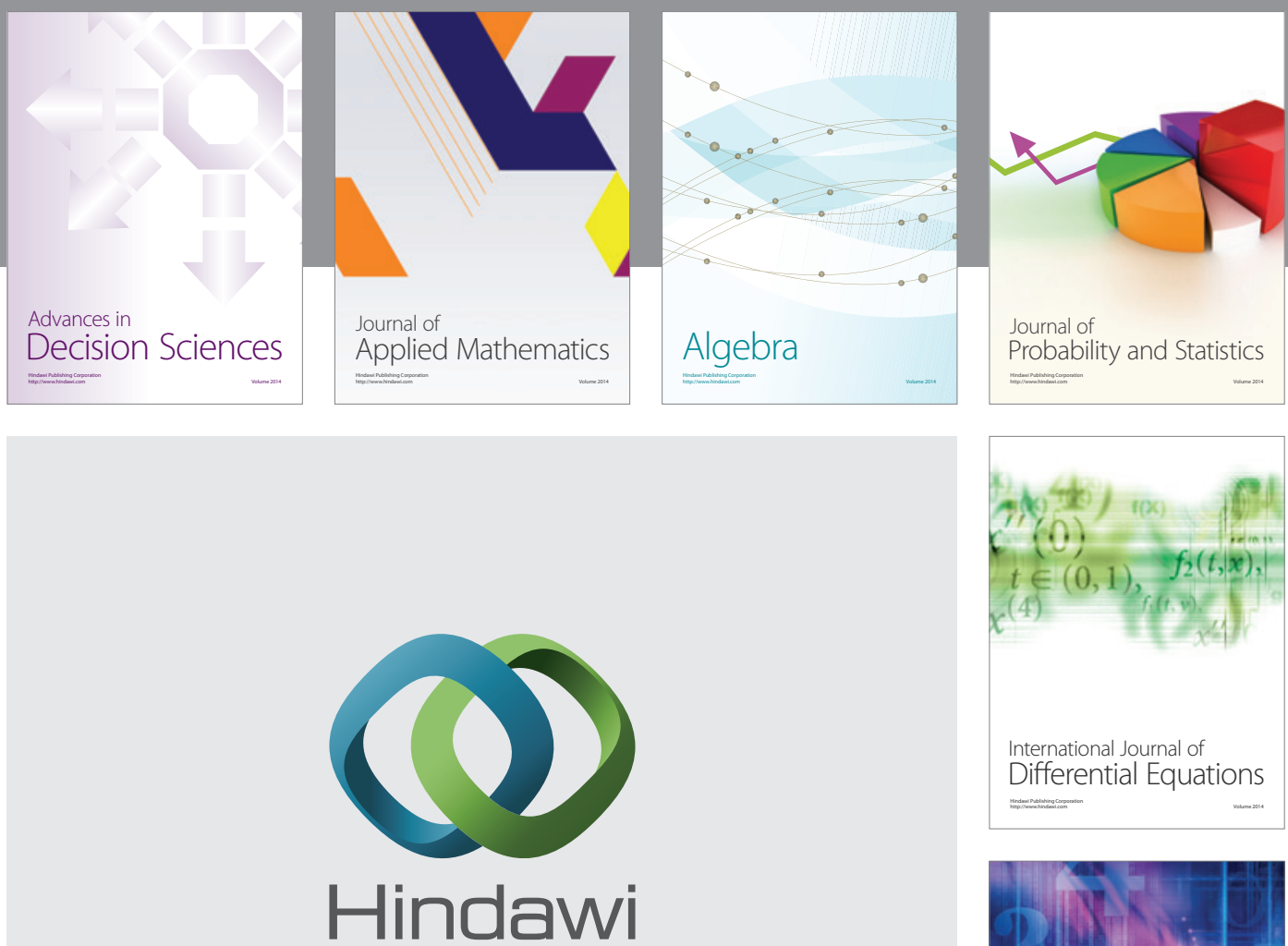

Submit your manuscripts at http://www.hindawi.com
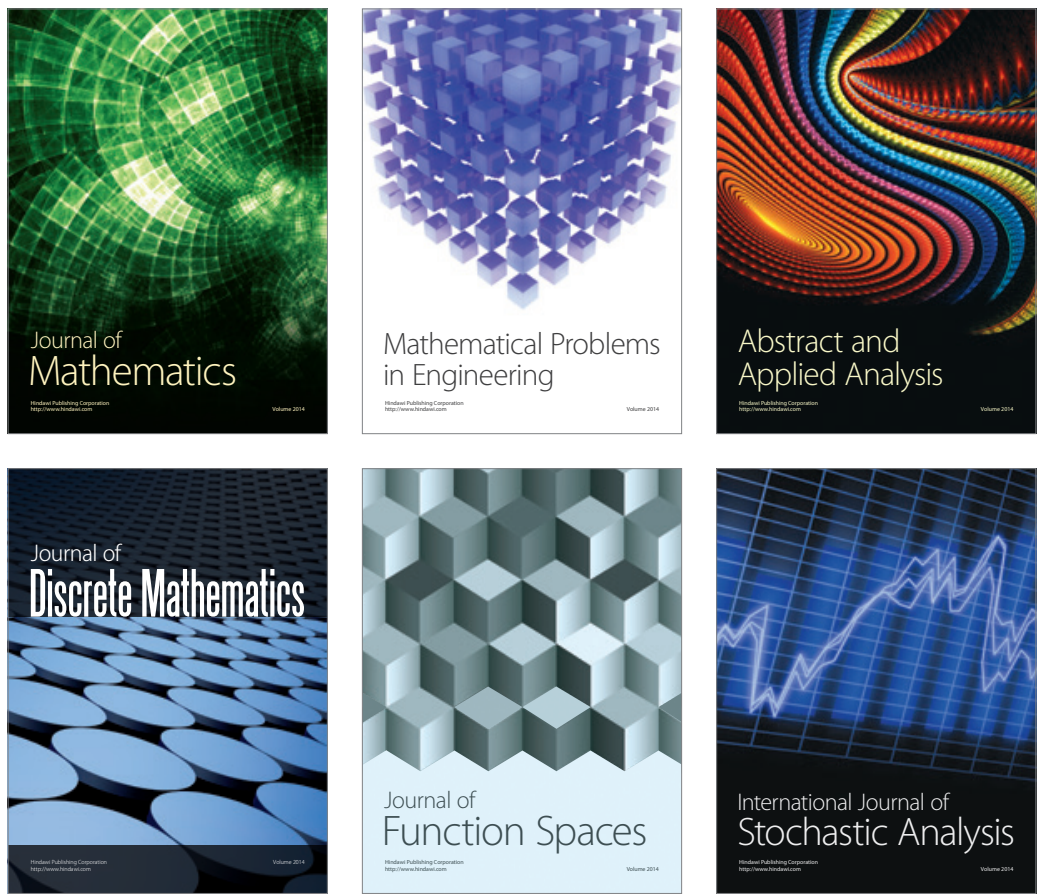

Journal of

Function Spaces

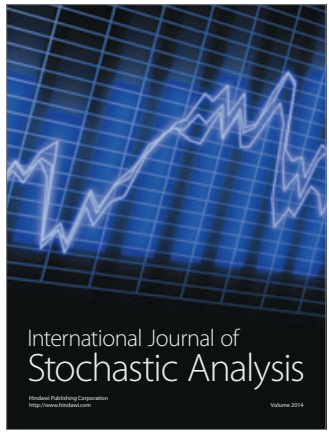

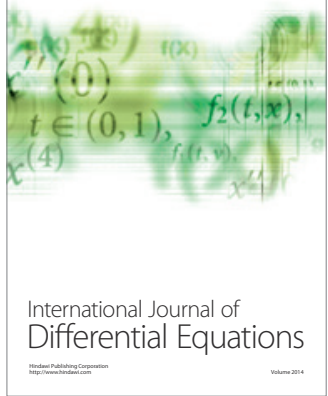
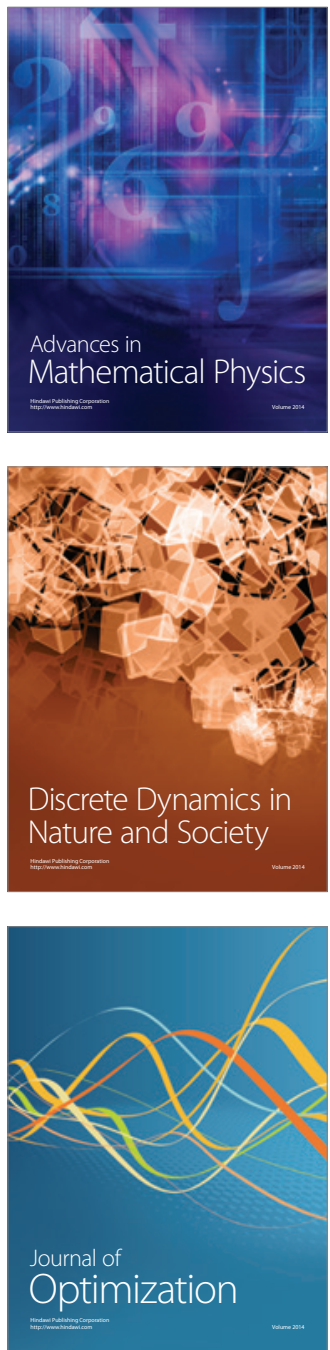